\title{
Staggered Phases in Diluted Systems with Continuous Spins
}

\author{
L. Chayes ${ }^{\star}$, R. Kotecký $\dot{y}^{\star \star}$, S. B. Shlosman ${ }^{\star \star \star}$ \\ Erwin Schrödinger Institute, Vienna, Austria
}

Received: 2 January 1997 / Accepted: 1 February 1997

Dedicated to the memory of Roland L'vovich Dobrushin

\begin{abstract}
We consider systems with continuous spins and annealed dilution. We show that, as in the discrete case, such systems often undergo a phase transition, which is manifested in the appearance of a staggered intermediate phase. In particular, these phases appear in systems such as the massive Gaussian model where there is no phase transition in the undiluted system.
\end{abstract}

\section{Introduction}

In this paper we continue the study, initiated in [CKS], of intermediate phases and first order transitions in annealed dilute spin systems. While in [CKS] we dealt with discrete spin systems, in the present paper we focus on systems with continuous spins.

It is normally the case that continuous spin systems are much harder to study than their discrete counterparts. The celebrated papers of Fröhlich, Simon, Spencer [FSS] and Fröhlich, Spencer [FS] were milestones in the rigorous understanding of the phase diagrams of these systems. It therefore came as a surprise to the authors that the problem of the intermediate phases for continuous annealed systems is not significantly harder than the discrete version. On the other hand, we have much less control over the phase diagrams of the continuous spin systems (in particular, systems with continuous symmetry) and of the properties of their different phases. This is reflected in the fact that our

\footnotetext{
* Permanent address: Department of Mathematics, UCLA, Los Angeles,CA 90095-1555, USA. E-mail: lchayes@math.ucla.edu

$\star \star$ On leave from Center for Theoretical Study, Charles University, Prague, and Department of Theoretical Physics, Charles University, V Holešovičkách 2, 18000 Praha 8, Czech Republic, E-mail: kotecky@cucc.ruk.cuni.cz; partly supported by the grants GAČR 202/96/0731 and GAUK 96/272.

$\star \star \star$ On leave from Department of Mathematics, University of California, Irvine, CA 92717, USA, E-mail: shlosman@math.uci.edu; partly supported by the NSF through grant DMS 9500958 and by the Russian Fund for Fundamental Research through grant 930101470.
} 
statements about phase diagrams of the dilute annealed systems with continuous spins are less complete than in the discrete spin case of [CKS].

The principal results of this work are all exhibited in the simplest (and best known) example of a continuous spin system: the $X Y$ model. Indeed, for brevity, all of the explicit proofs will concern the two-dimensional versions of this problem; we will be content with just stating general conditions under which analogous results can be established in other systems.

Thus consider the Hamiltonian

$$
H=-\sum_{i, j \in \mathbb{Z}^{2}}\left(\cos \left(\varphi_{i}-\varphi_{j}\right)-1\right) .
$$

Here the sum is over the nearest neighbors, and the $\varphi$ variables take values in the unit circle, which is identified with the segment $[0,2 \pi)$. The Hamiltonian $H_{s}$ of the site diluted version of this model is given by

$$
H_{s}=-\sum_{i, j \in \mathbb{Z}^{2}} n_{i} n_{j}\left(\cos \left(\varphi_{i}-\varphi_{j}\right)-1\right)-\mu \sum_{i \in \mathbb{Z}^{2}} n_{i}-\kappa \sum_{i, j \in \mathbb{Z}^{2}} n_{i} n_{j} .
$$

In the last equation the variables $n_{i}$ take on the values 0 or 1 , indicating the presence or absence of a particle at the site $i$. In the formula (1.1) the constant $(-1)$ is for convenience only - it can be omitted without loss of generality - this is not the case in (1.2); hence the introduction of the term with the parameter $\kappa$.

Our claim for the site diluted $X Y$ model concerns the existence of an intermediate phase within which there are two states that are characterized by the preferential occupation of the even/odd sublattices. This phase intercalates between the low temperature/high density regime and the high temperature/low density regime.

As in the discrete case, the existence of such a phase is due to entropic repulsion. While in the "large $q$-models" this is not difficult to understand, in the continuous case the origin of the effect is slightly more subtle. In the case, e.g. of the $X Y$ model, the explanation goes roughly as follows. If, at low temperatures, two particles are nearest neighbors, their spins have to be nearly aligned. In this case the available phase volume is $o(1)$ as $\beta \rightarrow \infty$. On the other hand if the sites are isolated, they may enjoy the full freedom of the circle; hence an effective repulsion is provided by the relative restrictions due to low temperature.

Our main result is that if the temperature is low (but not very low) the staggered phase indeed exists. Namely, in a region of intermediate temperature and chemical potential, there are (at least) the two staggered states. Moving out of the staggered phase towards the line $\mu=\infty$ (the uniform undiluted system), a portion of the phase boundary is a line of first order transitions. Here, the two staggered states coexist with the dense phase which may or may not be magnetically ordered depending on the details of the model. The rest of the phase boundary - the line between the staggered phases and the uniqueness regime - is, possibly, a line of higher order transitions. We will not not make any claims about these transitions.

The second sort of dilute systems we consider are the so called bond-dilute models. For the $X Y$ model, the relevant Hamiltonian is given by

$$
H_{b}=-\sum_{\langle i, j\rangle} n_{i, j}\left(\cos \left(\varphi_{i}-\varphi_{j}\right)-1\right)-\lambda \sum_{\langle i, j\rangle} n_{i, j},
$$

where the $n_{i, j}$ are bond occupation variables that take on the values zero or one and $\lambda$ is the bond fugacity. 
Here the results are also similar to the analogous problem for the discrete cases but, perhaps, more surprising for continuous spins. In particular, it will be established that extending from the point $\beta=\infty, \lambda=0$, there is a line of first order transitions across which the bond density - and the energy density - are discontinuous. In particular, note that for any configuration with a fixed number of bonds, as $T \rightarrow 0$ the energy is independent of the arrangement of bonds. Our results indicate that nevertheless, there is phase separation even at zero temperature.

\section{Site diluted models}

2.1. The two-dimensional $X Y$ model. As in [CKS], our analysis here relies heavily on the fact that all the systems we consider are reflection positive (RP). For two-dimensional systems with nearest neighbor interactions, we may use reflections in the lines

$$
\{x \pm y=k\}, \quad k=\ldots,-1,0,1, \ldots .
$$

The corresponding finite volume boxes that are invariant under such reflections are the two-dimensional tori

$$
\mathcal{T}_{N}=\left\{i=(x, y) \in \mathbb{Z}^{2}:|x \pm y| \leq N\right\},
$$

(or more precisely, the graphs thereof) where the standard identification of the boundary sites is assumed. Use of the tori $\mathcal{T}_{N}$ cuts down considerably on the amount of calculations that have to be performed. However, the above described advantages occur only for the two-dimensional case. In higher dimensions one has to consider reflections with respect to planes perpendicular to coordinate axes as was done in [CKS].

We denote by $H_{N}$ the restriction of the Hamiltonian $H_{s}$, given by (1.2), to the box $\mathcal{T}_{N}$. The partition function $\mathcal{Z}_{N, \beta}$ is given by

$$
\mathcal{Z}_{N, \beta}=\sum_{\substack{n_{i}=0,1 \\ i \in \mathcal{T}_{N}}} \int \exp \left\{-\beta H_{N}\left(n_{N}, \varphi_{N}\right)\right\} \prod_{i \in \mathcal{T}_{N} ; n_{i}=1} d \varphi_{i}
$$

This partition function serves as the normalization constant for the finite volume Gibbs state with periodic boundary conditions, $\langle-\rangle_{N, \kappa, \beta, \mu}$, which assigns to the configuration $\left(n_{N}, \varphi_{N}\right)$ a weight proportional to $\exp \left\{-\beta H_{N}\left(n_{N}, \varphi_{N}\right)\right\}$.

Let us now introduce the different possible infinite volume phases of the model, the existence of which are the crux of Theorem 2.1. They are denoted by $\langle-\rangle_{\kappa, \beta, \mu}^{o},\langle-\rangle_{\kappa, \beta, \mu}^{A}$ and $\langle-\rangle_{\kappa, \beta, \mu}^{B}$. The state $\langle-\rangle_{\kappa, \beta, \mu}^{o}$ is a small perturbation of the high density state of the $X Y$ model. In our context it is characterized by the high probability $\left\langle\chi_{i}^{1}\right\rangle_{\kappa, \beta, \mu}^{o}$ for any site to be occupied by a particle. (Here we have used $\chi_{i}^{1}$ to denote the indicator of the event $\left\{n_{N}, \varphi_{N} n_{i}=1\right\}$.) Moreover, if we introduce the indicator $\chi_{b}^{1,1}$ of the event that both sites of a bond $b$, (e.g. $b=[(0,0),(1,0)])$ are occupied by a particle, then its expected value $\left\langle\chi_{b}^{1,1}\right\rangle_{\kappa, \beta, \mu}^{o}$ is also close to one. The state $\langle-\rangle_{\kappa, \beta, \mu}^{A}$ describes the phase where the even sublattice is preferentially occupied and similarly $\langle-\rangle_{\kappa, \beta, \mu}^{B}$ for the odd sublattice. To characterize the state $\langle-\rangle_{\kappa, \beta, \mu}^{A}$ we introduce the indicator $\chi_{b}^{A}$ which is one when the even endpoint of the bond $b$ is occupied and the odd endpoint is vacant and vanishes otherwise. The indicator $\chi_{b}^{B}$ is described similarly with the roles of even and odd exchanged. The state $\langle-\rangle_{\kappa, \beta, \mu}^{A}$ is characterized by a value of $\left\langle\chi_{b}^{A}\right\rangle_{\kappa, \beta, \mu}^{A}$ close to one and similarly for the state $\langle-\rangle_{\kappa, \beta, \mu}^{B}$. We can now state: 
Theorem 2.1. Consider the two-dimensional site diluted $X Y$ model as described by the Hamiltonian in Eq. (1.2) with $\kappa>0$. There exist small numbers $\epsilon$ and $\kappa_{0}$ and, for every $\kappa<\kappa_{0}$, a region $F(\kappa) \subset \mathbb{R}$ so that for any $\mu \in F$ there exist inverse temperatures $\beta_{1}(\kappa, \mu), \beta_{2}(\kappa, \mu)$, and $\beta_{c}(\kappa, \mu) \in\left[\beta_{1}, \beta_{2}\right]$ such that:

i) For any $\beta \in\left[\beta_{c}, \beta_{2}\right]$ there exists a state \langle\rangle$_{\kappa, \beta, \mu}^{(o)}$, for which

$$
\left\langle\chi_{i}^{1}\right\rangle_{\kappa, \beta, \mu}^{(o)} \geq 1-\epsilon
$$

for every site $i$.

ii) For any $\beta \in\left[\beta_{1}, \beta_{c}\right]$ there exist two states \langle\rangle$_{\kappa, \beta, \mu}^{(A)}$ and \langle\rangle$_{\kappa, \beta, \mu}^{(B)}$ for which

$$
\left\langle\chi_{b}^{A}\right\rangle_{\kappa, \beta, \mu}^{(A)} \geq 1-\epsilon
$$

and

$$
\left\langle\chi_{b}^{B}\right\rangle_{\kappa, \beta, \mu}^{(B)} \geq 1-\epsilon
$$

respectively, for every bond $b$.

The proof of the above theorem via the RP technology requires certain estimates of the partition functions taken over subsets of configurations, which exhibit given pattern behavior. In our case of the two-dimensional $X Y$ model there are five such patterns. The first three of them are obtained by repeated reflections of the three characteristic patterns on the bond $b$, specified before Theorem 1 . One has to use the reflections in lines (2.1), until the pattern is disseminated to the whole $\mathcal{T}_{N}$. In this way we obtain the events:

$$
I_{N}^{A}=\left\{n_{N}, \varphi_{N}: n_{i}=1 ; i=(x, y) \in \mathcal{T}_{N}, x+y \text { even, } n_{i}=0 ; i \in \mathcal{T}_{N}, x+y \text { odd }\right\},
$$

- that the even sublattice is full, the analogous event $I_{N}^{B}$ - that the odd sublattice is full, and

$$
I_{N}^{o}=\left\{n_{N}, \varphi_{N}: n_{i}=1 \text { for all } i=(x, y) \in \mathcal{T}_{N}\right\},
$$

- that the whole box is filled by the particles. In addition we need the empty event - that all sites in $\mathcal{T}_{N}$ are vacant,

$$
I_{N}^{\emptyset}=\left\{n_{N}, \varphi_{N}: n_{i}=0 \text { for all } i=(x, y) \in \mathcal{T}_{N}\right\},
$$

and the event

$$
I_{N}^{c}=\left\{n_{N}, \varphi_{N}: n_{i}=0 \text { iff } i=(x, y) \in \mathcal{T}_{N}, x-y=4 k+1,\right\} .
$$

This last event is obtained from the elementary configuration $n_{(0,0)}=1, n_{(0,1)}=1$, $n_{(1,0)}=0$ on the union of two bonds, $b=[(0,0),(1,0)]$ and $b^{\prime}=[(0,0),(0,1)]$, by repeated reflections with respect to the lines (2.1), from which we exclude all lines of the form $\{x-y=2 k, \quad k=\ldots,-1,0,1, \ldots\}$. Elementary configurations of this type should appear whenever the two phases - ordered and staggered - touch each other; they form then the separating contour between the phases, which explains the superscript $c$ in our notation. We denote the indicator of the above elementary configuration by $\chi_{b, b^{\prime}}^{1,1,0}$.

We denote by $\chi_{N}^{A}, \chi_{N}^{B}, \chi_{N}^{o}, \chi_{N}^{\emptyset}$, and $\chi_{N}^{c}$ the indicators of the corresponding events $I_{N}^{A}, I_{N}^{B}, I_{N}^{o}, I_{N}^{\emptyset}$, and $I_{N}^{c}$. Finally, we define the partition functions, restricted to the above five events, by 


$$
\mathcal{Z}_{N, \beta}^{*}=\left\langle\chi_{N}^{*}\right\rangle_{N, \kappa, \beta, \mu} \mathcal{Z}_{N, \beta},
$$

where $*$ takes five values: $A, B, o, \emptyset$ and $c$.

The main step in proving Theorem 1 above consists of the following estimates:

Lemma 2.2. The partition functions (2.5) for the patterns $\emptyset, A, B$, o and c satisfy the following estimates:

$$
\begin{gathered}
\mathcal{Z}_{N, \beta}^{\emptyset}=1, \\
\left(\mathcal{Z}_{N, \beta}^{A}\right)^{1 /\left|\mathcal{T}_{N}\right|}=\left(\mathcal{Z}_{N, \beta}^{B}\right)^{1 /\left|\mathcal{T}_{N}\right|}=e^{\frac{1}{2} \beta \mu}(2 \pi)^{\frac{1}{2}}, \\
e^{\beta \mu} e^{2 \beta \kappa} \frac{e^{-1}}{\sqrt{\beta}} \leq\left(\mathcal{Z}_{N, \beta}^{o}\right)^{1 /\left|\mathcal{T}_{N}\right|} \leq e^{\beta \mu} e^{2 \beta \kappa} \frac{4}{\sqrt{\beta}}, \\
\left(\mathcal{Z}_{N, \beta}^{c}\right)^{1 /\left|\mathcal{T}_{N}\right|} \leq e^{\frac{3}{4} \beta \mu} e^{\beta \kappa}\left(\frac{4}{\sqrt{\beta}}\right)^{\frac{3}{4}},
\end{gathered}
$$

where $\left|\mathcal{T}_{N}\right|$ denotes the number of sites in $\mathcal{T}_{N}$ and various terms in (2.6)-(2.9) may be modified by multiplicative factors that tend to one as $N \rightarrow \infty$.

Proof. The identity (2.6) is obvious, and (2.7) is straightforward. To get the lower bound in (2.8) we restrict the range of integration to the product of arcs of length $\frac{1}{\sqrt{\beta}}$, each centered at the origin, i.e. to the set

$$
\left\{\left|\varphi_{i}\right|<\frac{1}{2 \sqrt{\beta}}, \quad i \in \mathcal{T}_{N}\right\} .
$$

We then replace the integrand by its minimal value and use the inequality $\cos \varphi-1 \geq$ $-\frac{\varphi^{2}}{2}$. To get the upper bound, we estimate the $\operatorname{sum} \sum_{i, j \in \mathcal{T}_{N},|i-j|=1}\left(\cos \left(\varphi_{i}-\varphi_{j}\right)-1\right)$ from above by restricting the range of summation from the set of all bonds to its subset, which forms a maximal tree. After that the integration can be done site by site, each contributing the factor of $\int \exp \{\beta(\cos \varphi-1)\} d \varphi$, which can be estimated from above with the help of the inequality:

$$
\cos \varphi-1 \leq-\left(\frac{\sqrt{2}}{\pi} \varphi\right)^{2}, \quad|\varphi| \leq \pi .
$$

The upper estimate (2.9) is obtained in the same way.

Proof of Theorem 2.1. The proof of this theorem goes in the manner that is customary for RP systems. We establish below that at very high $\beta$ and reasonable $\mu$ the ordered phase prevails, that for the same $\mu$ and lower $\beta$ 's the staggered phase prevails, and finally that their coexistence in space is ruled out in the whole interval of $\beta$ 's (contour estimate). We will also show that there are two different staggered phases by showing that their spatial coexistence is suppressed.

To implement this program we start with estimates of the expectations $\left\langle\chi_{b}^{A}\right\rangle_{N, \kappa, \beta, \mu}$ and $\left\langle\chi_{b}^{0,0}\right\rangle_{N, \kappa, \beta, \mu}$. By the chessboard estimate we have

$$
\left\langle\chi_{b}^{A}\right\rangle_{N, \kappa, \beta, \mu} \leq\left\langle\chi_{N}^{A}\right\rangle_{N, \kappa, \beta, \mu}^{\frac{1}{2\left|\mathcal{T}_{N}\right|}} \leq\left(\frac{\mathcal{Z}_{N, \beta}^{A}}{\mathcal{Z}_{N, \beta}^{o}}\right)^{\frac{1}{2\left|\mathcal{T}_{N}\right|}} \leq \sqrt{e} e^{-\frac{1}{4} \beta \mu} e^{-\beta \kappa}(2 \pi \beta)^{\frac{1}{4}},
$$

and 


$$
\left\langle\chi_{b}^{0,0}\right\rangle_{N, \kappa, \beta, \mu} \leq\left\langle\chi_{N}^{\emptyset}\right\rangle_{N, \kappa, \beta, \mu}^{\frac{1}{2\left|\mathcal{T}_{N}\right|}} \leq\left(\mathcal{Z}_{N, \beta}^{o}\right)^{-\frac{1}{2\left|\mathcal{T}_{N}\right|}} \leq \sqrt{e} e^{-\frac{1}{2} \beta \mu} e^{-\beta \kappa} \beta^{\frac{1}{4}},
$$

which is small for very large $\beta$ and positive $\mu$. Taking into account the identity

$$
\chi_{b}^{0,0}+\chi_{b}^{A}+\chi_{b}^{B}+\chi_{b}^{1,1}=1,
$$

these bounds show that at low temperatures almost all sites are occupied. Hence, one can choose $\beta_{2}=\infty$.

In the "staggered" region we estimate similarly $\left\langle\chi_{b}^{1,1}\right\rangle_{N, \kappa, \beta, \mu}$ and $\left\langle\chi_{b}^{0,0}\right\rangle_{N, \kappa, \beta, \mu}$. Again by the chessboard estimate

$$
\left\langle\chi_{b}^{1,1}\right\rangle_{N, \kappa, \beta, \mu} \leq\left\langle\chi_{N}^{o}\right\rangle_{N, \kappa, \beta, \mu}^{\frac{1}{2\left|\mathcal{T}_{N}\right|}} \leq\left(\frac{\mathcal{Z}_{N, \beta}^{o}}{\mathcal{Z}_{N, \beta}^{A}}\right)^{\frac{1}{2\left|\mathcal{T}_{N}\right|}} \leq 2 e^{\beta\left(\frac{1}{4} \mu+\kappa\right)}(2 \pi \beta)^{-\frac{1}{4}}
$$

and

$$
\left\langle\chi_{b}^{0,0}\right\rangle_{N, \kappa, \beta, \mu} \leq\left\langle\chi_{N}^{\emptyset}\right\rangle_{N, \kappa, \beta, \mu}^{\frac{1}{2\left|\mathcal{T}_{N}\right|}} \leq\left(\mathcal{Z}_{N, \beta}^{A}\right)^{-\frac{1}{2\left|\mathcal{T}_{N}\right|}} \leq e^{-\frac{1}{4} \beta \mu}(2 \pi)^{-\frac{1}{4}}
$$

It is clear, for $\kappa$ small enough, that we can find a $\mu \gtrsim 0$ and a $\beta_{1}=\beta_{1}(\mu, \kappa)$ with $\beta_{1} \gg 1$ such that for $\beta$ equal to (or slightly larger than) $\beta_{1}$, the right hand sides of Eqs. (2.13) and (2.14) are both small. To show that under these circumstances two staggered states coexist, we need a contour estimate. A site that belongs to more than one of the $A$-, $B$ - or $o$ - type bonds (a contour site) will be the center of a $\chi_{b, b^{\prime}}^{1,1,0}$-type event or be part of an empty bond. The latter are uniformly unlikely in the entire specified region, let us show that the same holds for the principal "contour term" $\left\langle\chi_{b, b^{\prime}}^{1,1,0}\right\rangle_{N, \kappa, \beta, \mu}$. This is readily accomplished:

$$
\begin{gathered}
\left\langle\chi_{b, b^{\prime}}^{1,1,0}\right\rangle_{N, \kappa, \beta, \mu} \leq\left\langle\chi_{N}^{c}\right\rangle_{N, \kappa, \beta, \mu}^{\frac{1}{T_{N} \mid}} \leq \frac{1}{\beta^{1 / 8}} \frac{4^{3 / 4} e^{1 / 2}}{(2 \pi)^{1 / 4}} \times \\
\times \frac{\frac{e^{-1 / 2}}{(2 \pi)^{1 / 4}} e^{\frac{1}{4} \beta \mu} e^{\beta \kappa} \frac{1}{\beta^{1 / 4}}}{\left[1+\left[\frac{e^{-1}}{(2 \pi)^{1 / 2}} e^{\frac{1}{2} \beta \mu} e^{2 \beta \kappa} \frac{1}{\beta^{1 / 2}}\right]^{\left|\mathcal{T}_{N}\right|}\right]^{\frac{1}{\left|\mathcal{T}_{N}\right|}}} \leq \frac{1}{\beta^{1 / 8}} \frac{4^{3 / 4} e^{1 / 2}}{(2 \pi)^{1 / 4}} .
\end{gathered}
$$

We used here the upper bound (2.9) as well as lower bounds (2.7) and (2.8) estimating first $\mathcal{Z}_{N, \beta} \geq \mathcal{Z}_{N, \beta}^{A}+\mathcal{Z}_{N, \beta}^{o}$.

All the required ingredients have now been assembled. It is clear that a finite region of any of the three competing phases is surrounded by contour sites. (In this case, the relevant notion of connectivity for the contours is $*$-connectedness.) It follows that for all $\beta$ in $\left[\beta_{1}, \infty\right)$, the contours are damped exponentially with their length. In the region where the ordered indicator has small expectation, i.e. $\beta \gtrsim \beta_{1}$ this implies the existence of the two staggered states. For $\beta$ large we of course have the ordered state. Applying Lemma (2.4) from [CKS], we may conclude that there is a $\beta_{c} \in\left(\beta_{1}, \infty\right)$, where the two staggered states coexist with an ordered state.

Remark. The fact that Theorem 2.1 was proved for $\kappa>0$ allowed us to easily demonstrate that the staggered phase does not survive down to zero temperature. In fact, for $\beta \gg 1, \kappa$ fixed and $\mu$ allowed to vary, the system undergoes a first order phase transition near $\mu=-d \kappa$, where the (almost fully) occupied state coexists with a state in which nearly every site is vacant. Such results were established in [CKS] in the discrete cases; here the proof is nearly identical. 
2.2. The general case. The results from the preceding section can be extended to a general class of nearest neighbor site diluted models on $\mathbb{Z}^{d}, d \geq 2$. Namely, we consider a model with spins $\varphi$ taking values in a Riemannian manifold $\mathcal{S}$ equipped with an a priori Borel measure $\mu(d \varphi)$. The metric on $\mathcal{S}$ will be denoted by $\rho(\cdot, \cdot)$, the corresponding Riemann measure is $d \varphi$, and $\mu(d \varphi)$ is supposed to be absolutely continuous with respect to $d \varphi$, with a continuous density, such that $\int d \mu<\infty$. The Hamiltonian on the torus $\mathcal{T}_{N}$ has the form

$$
H_{N}\left(n_{N}, \varphi_{N}\right)=\sum_{\langle i, j\rangle} n_{i} n_{j} U\left(\varphi_{i}, \varphi_{j}\right)-\mu \sum_{i} n_{i}-\kappa \sum_{\langle i, j\rangle} n_{i} n_{j} .
$$

Here $\mu, \kappa$, and the occupation variables $n_{i} \in\{0,1\}$ play the same role as in the particular case (1.2) of $X Y$ model. For $\varphi, \psi \in \mathcal{S}$, it is supposed that the interaction $U(\varphi, \psi)$ satisfies the following conditions:

- $U(\cdot, \cdot)$ is measurable and $U(\varphi, \psi) \geq 0$ for each $\varphi, \psi \in \mathcal{S}$.

- There exist $\varphi_{0} \in \mathcal{S}$ such that $U\left(\varphi_{0}, \varphi_{0}\right)=0$.

- The minimum of $U(\cdot, \cdot)$ at the point $\varphi_{0}$ is essential: Namely, using $O_{r}(\varphi)$, for any $\varphi \in \mathcal{S}$, to denote the neighborhood $O_{r}(\varphi)=\{\psi \in \mathcal{S} \rho(\varphi, \psi) \leq r\}$, we suppose that for some $k>0$ we have

$$
\max _{\varphi, \psi \in O_{r}\left(\varphi_{0}\right)} U(\varphi, \psi) \leq C_{1} r^{k}
$$

for $r$ small enough.

- Attractiveness: for any $\varphi, \psi \in \mathcal{S}$ we have

$$
U(\varphi, \psi) \geq C_{2} \rho(\varphi, \psi)^{k} .
$$

Introducing the indicators $\chi_{i}^{1}, \chi_{b}^{A}$, and $\chi_{b}^{B}$ in the same way as in the case of $X Y$ model, we get the anticipated generalization. Its proof is a rather straightforward extension of the proof of Theorem 2.1 above applying the version of RP used in the proof of Theorem 3.1 in [CKS].

Theorem 2.3. Consider the site diluted model as described by the Hamiltonian $H_{N}$ in Eq. (2.16) with $\kappa>0$ and with interaction $U$ satisfying the conditions above. There exist small numbers $\epsilon$ and $\kappa_{0}$ and, for every $\kappa<\kappa_{0}$, a region $F(\kappa) \subset \mathbb{R}$ so that for any $\mu \in F$ there exist inverse temperatures $\beta_{1}(\kappa, \mu), \beta_{2}(\kappa, \mu)$, and $\beta_{c}(\kappa, \mu) \in\left(\beta_{1}, \beta_{2}\right)$ such that:

i) For any $\beta \in\left[\beta_{c}, \beta_{2}\right]$ there exists a state \langle\rangle$_{\kappa, \beta, \mu}^{(o)}$, for which

$$
\left\langle\chi_{i}^{1}\right\rangle_{\kappa, \beta, \mu}^{(o)} \geq 1-\epsilon
$$

for every site $i$.

ii) For any $\beta \in\left[\beta_{1}, \beta_{c}\right]$ there exist two states \langle\rangle$_{\kappa, \beta, \mu}^{(A)}$ and \langle\rangle$_{\kappa, \beta, \mu}^{(B)}$ for which

$$
\left\langle\chi_{b}^{A}\right\rangle_{\kappa, \beta, \mu}^{(A)} \geq 1-\epsilon
$$

and

$$
\left\langle\chi_{b}^{B}\right\rangle_{\kappa, \beta, \mu}^{(B)} \geq 1-\epsilon
$$

respectively, for every bond $b$. 
Proof (sketch). The basic ideas have been spelled out in the above mentioned resources; the major distinctions are technical. We consider elementary hypercubes of side 2 and assign patterns $A, B, \emptyset$, etc. to these cubes. Any cube that does not fall into such a pattern is part of a contour. Estimates for the partition functions associated with these cubes are straightforward given the stated conditions. For example, the ordered partition function per site has upper and lower bounds of the form $e^{\beta \mu} e^{d \beta \kappa} \beta^{-w}$ with $w>0$ depending on the details of the internal spin-space. Contour cubes are then listed (or classified) and controlled with a chessboard estimate. In general, each constrained site in the contour pattern costs a factor of $\beta^{-w}$ and there are not enough favorable bonds to compensate; the details are straightforward but tedious. The upshot is that all these objects are uniformly suppressed by inverse powers of $\beta$. For $\kappa$ sufficiently small and $\mu>0$ we find a large $\beta_{1}$, where staggered order dominates and for $\beta \gg 1$, any state is a perturbation of the fully occupied state. The claimed results follows, mutatis mutandis, from previous derivations.

Remark. It is evident that this theorem applies even in cases where the undiluted models does not undergo a phase transition. Thus, for example, one can consider Gaussian lattice field $\varphi_{i} \in \mathbb{Z}^{\nu}$ with the Hamiltonian

$$
H_{N}\left(n_{N}, \varphi_{N}\right)=\sum_{\langle i, j\rangle} n_{i} n_{j}\left[\left(\varphi_{i}-\varphi_{j}\right)^{2}+m^{2}\left(\left(\varphi_{i}\right)^{2}+\left(\varphi_{j}\right)^{2}\right)\right]-\mu \sum_{i} n_{i}-\kappa \sum_{\langle i, j\rangle} n_{i} n_{j},
$$

and use Theorem 2.2 to show the existence of staggered phase.

\section{Bond diluted models}

3.1. The two-dimensional $X Y$ model. We consider $\mathcal{T}_{N}$ as defined in Eq. (2.2) and the restriction $H_{N}$ of the Hamiltonian in Eq. (1.3) to $\mathcal{T}_{N}$. The partition function, here denoted by $Z_{N, \beta}$, is given by

$$
\begin{gathered}
Z_{N, \beta, \lambda}=\sum n_{i, j}=0,1 \\
\langle i, j\rangle \in \mathcal{T}_{N} \int \exp \left\{-\beta H_{N}\left(\underline{n}_{N}, \phi_{N}\right)\right\} \prod_{i \in \mathcal{T}_{N}} d \phi_{i},
\end{gathered}
$$

where $\underline{n}_{N}$ denotes a bond configuration on $\mathcal{T}_{N}$. Similarly, $H_{N}$ is used to define the finite volume states $\langle-\rangle_{N, \lambda, \beta}$ on $\mathcal{T}_{N}$.

Here, there are only two relevant infinite volume states: $\langle-\rangle_{\lambda, \beta}^{(o)}$ and $\langle-\rangle_{\lambda, \beta}^{(\emptyset)}$ representing states with nearly all full and nearly all empty bond configurations. For this problem, we consider the variable $n_{i, j}$ itself: $\langle-\rangle_{\lambda, \beta}^{(o)}$ is distinguished by a value of $\left\langle n_{i, j}\right\rangle_{\lambda, \beta}^{(o)}$ that is close to one, while $\left\langle n_{i, j}\right\rangle_{\lambda, \beta}^{(\emptyset)}$ is close to zero.

Theorem 3.1. Consider the two-dimensional bond-diluted $X Y$-model as described by the Hamiltonian in Eq. (1.3). Then for all $\beta$ sufficiently large, there is $a \lambda_{c}(\beta)$ and a small number $\epsilon$ such that at $\left(\beta, \lambda_{c}(\beta)\right)$, there are two coexisting infinite volume states $\langle-\rangle_{\lambda_{c}, \beta}^{(o)}$ and $\langle-\rangle_{\lambda_{c}, \beta}^{(\emptyset)}$ with

$$
\left\langle n_{i, j}\right\rangle_{\lambda_{c}, \beta}^{(o)} \geq 1-\epsilon
$$

and

$$
\left\langle n_{i, j}\right\rangle_{\lambda_{c}, \beta}^{(\emptyset)} \leq \epsilon
$$


Proof. Denoting by $\chi_{N}^{o}$ and $\chi_{N}^{\emptyset}$ the indicators of the events that all bonds in $\mathcal{T}_{N}$ are occupied or, respectively, vacant, and $Z_{N, \beta, \lambda}^{*}=Z_{N, \beta, \lambda}\left\langle\chi^{*}\right\rangle_{N, \lambda, \beta}$ with $*=o$ or $\emptyset$, we start with the observation that

$$
\left(Z_{N, \beta, \lambda}^{\emptyset}\right)^{1 /\left|\mathcal{T}_{N}\right|}=2 \pi
$$

and

$$
e^{2 \beta \lambda} e^{-1} \frac{1}{\sqrt{\beta}} \leq\left(Z_{N, \beta, \lambda}^{\emptyset}\right)^{1 /\left|\mathcal{T}_{N}\right|} \leq e^{2 \beta \lambda} \frac{4}{\sqrt{\beta}} .
$$

Indeed, the first part of Eq. 3.2 is trivial and, after pulling out a factor of $e^{\beta \lambda}$ for each bond, the second part is just the estimates performed for Eq. (2.8) in the proof of Lemma 2.2 . It is thus clear that as $\lambda \rightarrow \pm \infty$, fully occupied/fully vacant states are predominant.

Next we consider the contour term. Here, the relevant contour piece occurs when two bonds, $b=\langle i, j\rangle$ and $b^{\prime}=\left\langle i, j^{\prime}\right\rangle$ on the corners of a square satisfy $n_{i, j}=1$ and $n_{i, j^{\prime}}=0$. Let $\chi_{b, b^{\prime}}^{1,0}=n_{b}\left(1-n_{b^{\prime}}\right)$ denote the indicator for this event and let $\chi_{N}^{c}$ denote the indicator for the event that the torus is covered by the bond pattern that is defined by Eq. (2.4). Finally, let $Z_{N, \beta, \lambda}^{c}$ denote the partition function associated with the indicator $\chi_{N}^{c}$. Then, by the chessboard estimate, we have

$$
\left\langle\chi_{b, b^{\prime}}^{1,0}\right\rangle_{N, \beta} \leq\left[Z_{N, \beta, \lambda}^{c} / Z_{N, \beta, \lambda}\right]^{1 /\left|\mathcal{T}_{n}\right|} .
$$

We claim (modulo terms that tend to one as $N$ gets large) that $\left(Z_{N, \beta, \lambda}^{c}\right)^{1 /\left|\mathcal{T}_{N}\right|} \leq$ $\left[(2 \pi)^{1 / 4} e^{\beta \lambda}[4 / \sqrt{\beta}]^{3 / 4}\right]$. Indeed, this is the same sort of estimate as the bound (2.9) and may be proved as in Lemma 2.2.

To finish this proof, it remains to show that this contour term is uniformly small if $\beta$ is large. We follow the reasoning that was used to derive the bound in Eq. (2.15) and arrive at

$$
\left\langle\chi_{b, b^{\prime}}^{1,0}\right\rangle_{N, \beta} \leq \frac{1}{\beta^{1 / 8}} \frac{4^{3 / 4} e^{1 / 2}}{(2 \pi)^{1 / 4}}
$$

which is manifestly small if $\beta$ is large. By the previously used arguments, this shows that for some value of $\lambda$, there is phase coexistence.

3.2. The general case. Finally, we consider bond dilute problems with continuous spins in a more general setting. Thus we write

$$
H_{N}\left(n_{N}, \varphi_{N}\right)=\sum_{\langle i, j\rangle} n_{i, j} U\left(\varphi_{i}, \varphi_{j}\right)-\lambda \sum_{\langle i, j\rangle} n_{i, j}
$$

for the Hamiltonian restricted to the $d$-dimensional torus of scale $N$. It is supposed that the space $\mathcal{S}$ and the potential $U$ satisfy the conditions spelled out just prior to Theorem 2.3; the quantities $\lambda$ and $n_{i, j}$ have the same meaning as in the $X Y$-case. The following can be established:

Theorem 3.2. Consider the d-dimensional bond-diluted models as described by the Hamiltonian in Eq. (3.5) and satisfying the subsequently stated conditions. Then the results stated in Theorem 3.1 hold in these cases.

Proof. The necessary modifications are similar to those required in the generalization of the site-diluted cases; the relevant portion of [CKS] is Theorem 4.2. 


\section{References}

[CKS] Chayes, L., Kotecký, R., and Shlosman, S.: Aggregation and Intermediate Phases in Dilute Spin Systems. Comm. Math. Phys. 171, 203-232 (1995)

[FS] Fröhlich, J. and Spencer, T.: The Kosterlitz-Thouless Transition in Two-dimensional Abelian Systems and the Coulomb Gas. Comm. Math. Phys. 81, 527-602 (1981)

[FSS] Fröhlich, J., Simon, B., and Spencer, T.: Infrared Bounds, Phase Transitions and Continuous Symmetry Breaking. Comm. Math. Phys. 50, 79-95 (1976)

Communicated by Ya. G. Sinai 